\title{
SCIENTIFIC MOTIVATION FOR ADM/AEOLUS MISSION
}

\author{
Erland Källén
}

\section{ECMWF, Shinfield Park, Reading, RG2 9AX, United Kingdom, erland.kallen@ecmwf.int}

\begin{abstract}
The ADM/Aeolus wind lidar mission will provide a global coverage of atmospheric wind profiles. Atmospheric wind observations are required for initiating weather forecast models and for predicting and monitoring long term climate change. Improved knowledge of the global wind field is widely recognised as fundamental to advancing the understanding and prediction of weather and climate. In particular over tropical areas there is a need for better wind data leading to improved medium range (3-10 days) weather forecasts over the whole globe.
\end{abstract}

\section{INTRODUCTION}

Together with temperature, pressure and humidity, wind is one of the basic variables describing the state of the atmosphere. Atmospheric wind observations are required for initiating weather forecast models and for predicting and monitoring long term climate change. Improved knowledge of the global wind field is widely recognised as fundamental to advancing the understanding and prediction of weather and climate [1]. The present in-situ and surface based global observing system for winds has large gaps, in particular over ocean areas and in the tropics. It includes the radiosonde network, commercial aircraft, wind profilers, Doppler radars and surface stations. Winds can also be measured from satellites, cloud track winds from imagers and scatterometers measuring ocean surface winds are the two dominant observation sources. All wind observing systems suffer from a number of limitations, the most important are a lack of global coverage and accuracy limitations. Only the radiosondes and the wind profilers provide vertical wind profiles, most of the other observations are single level or vertically averaged over deep layers. Radiosondes and wind profilers are primarily located over inhabited areas of the northern hemisphere, there are very few observations in the tropics and almost none over the vast ocean areas in the southern hemisphere.
The ADM/Aeolus mission will provide a global coverage of atmospheric wind profiles, urgently needed to enhance the quality of weather forecasts and improve climate analyses [2]. Of particular interest is the potential to improve analysis of tropical weather systems, both in terms of accuracy of the initial state for numerical weather prediction and in terms of tropical circulation monitoring in climate analyses. Improvements in the tropics will lead to improved weather forecasts in mid-latitudes as initial state errors in the tropics propagate to Europe, Asia and North America within a week and thus affect weather forecast quality in the medium range (3-10 days). There are examples of very poor six day forecasts over Europe that can be traced back to initial state errors in the tropics [3]. The wind profiles will also help to better define smaller scale circulation systems in mid-latitudes, examples are atmospheric fronts and polar lows. In the upper troposphere and lower stratosphere (UTLS) the wind profiles will enhance circulation analyses that helps to improve weather prediction and better understand climate change dynamics in the UTLS region.

\section{METHODOLOGY}

The Aeolus Doppler wind lidar instrument will measure line-of-sight winds in the troposphere and lower stratosphere. Only one horizontal wind component is measured, predominantly in the zonal direction. Aeolus is a polar orbiting satellite flying in a sun-synchronous dawn-dusk orbit at about $350 \mathrm{~km}$ height. Wind profile measurements will be provided with a horizontal resolution of about $80 \mathrm{~km}$ and a vertical resolution ranging from about 500 meters close to the surface up to about $2 \mathrm{~km}$ in the lower stratosphere. To obtain the full, two component, horizontal wind field the Aeolus line-of-sight wind data must be assimilated in a global NWP system. This will be undertaken at ECMWF who will be responsible for providing level $2 \mathrm{C}$ wind products using the operational NWP assimilation system. In addition 
cloud and aerosol information will be provided using information on lidar scattering and backscatter to extinction ratios. Comprehensive information about the Aeolus mission can be found in the Aeolus science report published by ESA [4] as well as in other presentations at the ILRC28 conference.

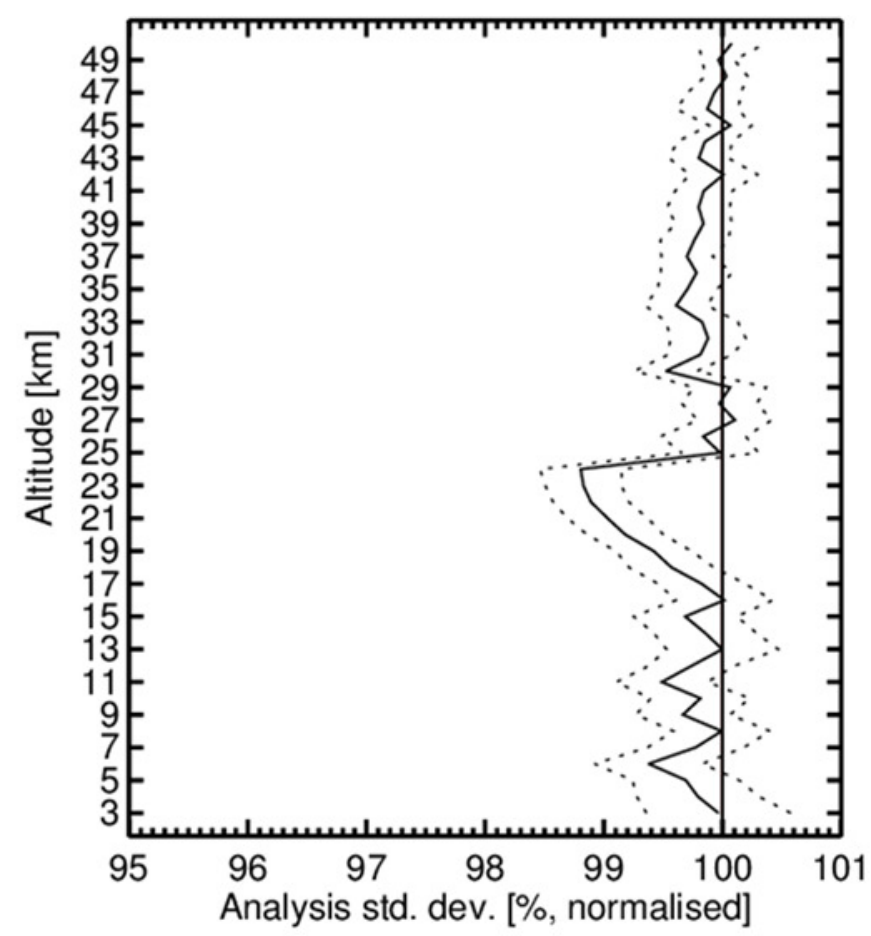

Figure 1 Difference between two assimilation experiments of the fit between analysis (left hand plot) and background (right hand plot) to observed GPSRO bending angles in the tropics.

\section{TROPICAL ASSIMILATION}

The atmospheric motion and thermodynamic structures in the tropics are quite different from the ones found in midlatitudes. Due to the influence of the rotation of the Earth there is a quasi-stationary balance between temperature, pressure and wind fields in the extra-tropics, i.e. north and south of 30 degrees latitude. Within the tropics the influence of the Earth's rotation on this balance is very different, instead of a quasistationary local balance between temperature, pressure and wind we find large scale wave structures that propagate slowly along the equatorial belt. Close to the equator these structures are defined by the wind field, temperature and pressure only exhibit small variations and the winds determine the variations. Further away from the equator temperature, pressure and wind become more balanced but they are linked to the equatorial flow as the large scale wave structures cover several thousands of kilometers [5]. With more wind data we are better able to define tropical wave structures, only mass

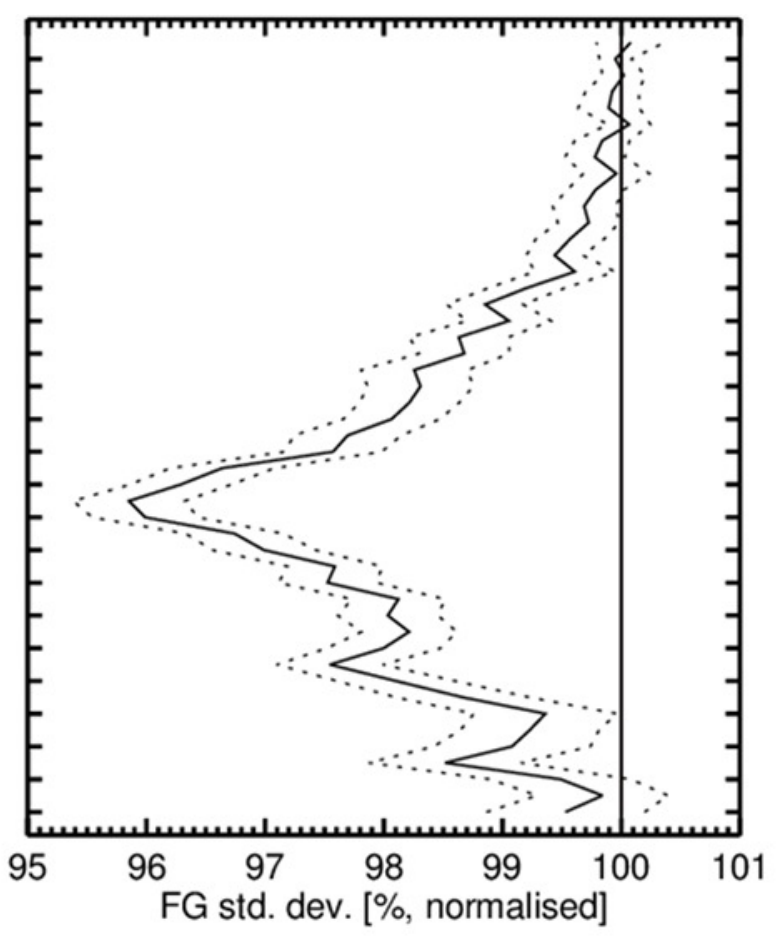

field data (temperature and humidity) is not sufficient to constrain the wind field. Wind profile data from Aeolus will improve the quality of tropical analyses and reduce initial state errors in numerical weather prediction [6]. Synthetic Aeolus data impact experiments have shown that adding Aeolus data to the ECMWF prediction systems gives about the same positive impact on forecast quality as radiosonde data gives us today [7]. In particular we find cases where medium range forecasts failures over Europe can be traced back to initial state errors in the tropics [3]. Another observation system simulation experiment also illustrates the direct impact of line-of-sight wind observations in reducing short range tropical forecast errors [8].

In addition to providing better defined wind fields in the tropics Aeolus data will also improve the assimilation of mass field data in the tropics. Using the ECMWF assimilation system and performing assimilation of mass field data with 
and without line-of-sight wind data the fit of analyses and short range forecasts to observed radio occultation (GPSRO) data in the tropics demonstrates the beneficial impact of wind data. In fig 1 we show the relative change of GPSRO bending angle fits to observations comparing one experiment with all conventional wind data removed and the other with line-of-sight wind data included to simulate the upcoming Aeolus wind profiling mission. A reduction of the standard deviation means that the fit improves with more wind data. In figure 1 there is a marked reduction in the UTLS region indicating the importance of wind information in this region.

The Aeolus mission will also provide information about clouds and aerosols from lidar backscatter intensities. This data will be useful for assimilating aerosol information into NWP systems and contribute to improving atmospheric composition monitoring and forecasting.

\section{CONCLUSIONS}

WMO co-ordinated assessments of observational needs for numerical weather prediction have repeatedly highlighted the primary importance of improved wind observations. The ADM/Aeolus wind profiles will have a significant impact on the quality of weather forecasts and climate analyses. In particular

\section{ACKNOWLEDGEMENTS}

Michael Rennie is gratefully acknowledged for providing fig 1.

\section{References}

[1] Baker, W. E., Atlas, R., Cardinali, C., Clement, A., Emmitt, G. D., Gentry, B. M., Hardesty, R M., Källén, E., Kavaya, M. J., Langland, R., Ma, Z., Masutani, M., McCarty, M., Pierce, R.B., $\mathrm{Pu}, \mathrm{Z}$., Riishojgaard, L. P. R., Ryan, J., Tucker, S., Weissman, M. and Yoe, J. G., 2014: Lidarmeasured wind profiles: The missing link in the global observing system. Bull. Amer. Met. Soc., 95, 543-564.

[2] Stoffelen, A., Pailleux, J., Källén, E., Vaughan, J. M., Isaksen, L., Flamant, P., Wergen, W., Andersson, E., Schyberg, H. and Meynart, R., 2005. The Atmospheric Dynamics Mission for global wind field measurement. Bulletin of the American Meteorological Society, 86, 73-87.

[3] Magnusson, L., 2017: Diagnostic methods for understanding the origin of forecast errors. ECMWF Technical Memorandum no. 796, pp 26.

[4] ESA, 2008: ADM-Aeolus Science report. ESA report SP-1311, pp 121.

[5] Žagar, N., N. Gustafsson and E. Källén, 2004: Dynamical response of equatorial waves in fourdimensional variational data assimilation. Tellus. 56A, 29-46.

[6] Žagar, N., 2004. Assimilation of equatorial waves by line-of-sight wind observations. Journal of the Atmospheric Sciences, 61, 18771893.

[7] Tan, D. G. and E. Andersson, 2005. Simulation of the yield and accuracy of wind profile measurements from the Atmospheric Dynamics Mission (ADM-Aeolus). Quarterly Journal of the Royal Meteorological Society, 131, 17371757.

[8] Horányi, A., Cardinali, C., Rennie, M. and Isaksen, L., 2015. The assimilation of horizontal line-of-sight wind information into the ECMWF data assimilation and forecasting system. Part I: The assessment of wind impact. Quarterly Journal of the Royal Meteorological Society, 141, 1223-1232. 